\title{
Outer boundary conditions for evolving cool white dwarfs ${ }^{\star}$ (Research Note)
}

\author{
R. D. Rohrmann ${ }^{1, \star \star}$, L. G. Althaus ${ }^{2, * *}$, E. García-Berro ${ }^{3,4}$, A. H. Córsico ${ }^{2, * *}$, and M. M. Miller Bertolami2 ${ }^{2, * *}$ \\ 1 Instituto de Ciencias Astronómicas, de la Tierra y del Espacio (CONICET-UNSJ), Av. España 1512 (sur), 5400 San Juan, Argentina \\ e-mail: rohr@icate-conicet.gob.ar \\ 2 Facultad de Ciencias Astronómicas y Geofísicas, Universidad Nacional de La Plata, Paseo del Bosque s/n, 1900 La Plata, Argentina \\ e-mail: althaus@fcaglp.unlp.edu.ar \\ 3 Departament de Física Aplicada, Universitat Politècnica de Catalunya, c/Esteve Terrades 5, 08860 Castelldefels, Spain \\ e-mail: garcia@fa.upc.edu \\ ${ }^{4}$ Institute for Space Studies of Catalonia, c/Gran Capità 2-4, Edif. Nexus 104, 08034 Barcelona, Spain
}

Received 27 March 2012 / Accepted 4 September 2012

ABSTRACT

\begin{abstract}
Context. White dwarf evolution is essentially a gravothermal cooling process, which, for cool white dwarfs, depends on the treatment of the outer boundary conditions.

Aims. We provide detailed outer boundary conditions that are appropriate to computing the evolution of cool white dwarfs by employing detailed nongray model atmospheres for pure hydrogen composition. We also explore the impact on the white dwarf cooling times of different assumptions for energy transfer in the atmosphere of cool white dwarfs.

Methods. Detailed nongray model atmospheres were computed by considering nonideal effects in the gas equation of state and chemical equilibrium, collision-induced absorption from molecules, and the Lyman $\alpha$ quasi-molecular opacity. We explored the impact of outer boundary conditions provided by updated model atmospheres on the cooling times of 0.60 and $0.90 M_{\odot}$ white dwarf sequences. Results. Our results show that the use of detailed outer boundary conditions becomes relevant for effective temperatures lower than $5800 \mathrm{~K}$ for sequences with $0.60 M_{\odot}$ and $6100 \mathrm{~K}$ with $0.90 M_{\odot}$. Detailed model atmospheres predict ages that are up to $\approx 10 \%$ shorter at $\log \left(L / L_{\odot}\right)=-4$ when compared with the ages derived using Eddington-like approximations at $\tau_{\text {Ross }}=2 / 3$. We also analyze the effects of various assumptions and physical processes that are relevant in the calculation of outer boundary conditions. In particular, we find that the Ly $\alpha$ red wing absorption does not substantially affect the evolution of white dwarfs.

Conclusions. White dwarf cooling timescales are sensitive to the surface boundary conditions for $T_{\text {eff }} \lesssim 6000 \mathrm{~K}$. Interestingly enough, nongray effects have few consequences on these cooling times at observable luminosities. In fact, collision-induced absorption processes, which significantly affect the spectra and colors of old white dwarfs with hydrogen-rich atmospheres, have no noticeable effects on their cooling rates, except throughout the Rosseland mean opacity.
\end{abstract}

Key words. stars: evolution - stars: interiors - white dwarfs

\section{Introduction}

An accurate assessment of the rate at which white dwarfs cool down is a fundamental issue because these stars can be used as independent and accurate age indicators. As a matter of fact, white dwarfs are the most common endpoint of stellar evolution - see, for instance, Althaus et al. (2010a) for a recent review - and as such are valuable in constraining several properties of a wide variety of stellar populations, including globular and open clusters (Von Hippel \& Gilmore 2000; Hansen et al. 2007; Winget et al. 2009; García-Berro et al. 2010). Additionally, they can be used to place constraints on elementary particles, such as axions (Isern et al. 1992; Córsico et al. 2001; Isern et al. 2008) and neutrinos (Winget et al. 2004), or on alternative theories of gravitation (García-Berro et al. 1995, 2011). These and other potential applications require detailed and precise knowledge of the main physical processes that control their evolution. A key

\footnotetext{
* Table with the outer boundary conditions is only available at the CDS via anonymous ftp to

cdsarc.u-strasbg.fr $(130.79 .128 .5)$ or via

http://cdsarc.u-strasbg.fr/viz-bin/qcat?]/A+A/546/A119

$\star \star$ Member of CONICET, Argentina.
}

ingredient is the energy transfer in the atmospheric and subatmospheric layers that control their cooling (Mestel 1952). Once convection reaches the outer edge of the degenerate core in low-luminosity white dwarfs - so-called convective coupling the cooling becomes strongly tied to the treatment of the outer boundary conditions (Böhm \& Grenfell 1973). Consequently, an accurate assessment of the cooling rate at low luminosities requires using detailed model atmospheres (Hansen 1998; Salaris et al. 2000; Serenelli et al. 2001).

The treatment of the energy transfer in white dwarf atmospheres is a difficult task that involves solving the equations of radiative transfer coupled to convection, in a highly nonideal gas regime where several molecular and quasi-molecular processes have to be considered. This implies a high degree of sophistication for the calculations, especially at very low luminosities. The importance of using detailed boundary conditions was first addressed by Hansen (1998, 1999). Over the years, detailed model atmospheres have been developed that include a complete treatment of energy absorption processes such as collision-induced opacity (Bergeron et al. 1991; Saumon \& Jacobson 1999; Rohrmann 2001) and the Lyman $\alpha$ wing absorption (Kowalski \& Saumon 2006; Rohrmann et al. 2011). 
Here we provide detailed boundary conditions that allow consistently computation of the evolution of cool white dwarfs with pure hydrogen atmospheres. These boundary conditions are provided in the form of tables for a wide range of surface gravities and effective temperatures. In the following sections we describe the model atmospheres (Sect. 2) and the evolutionary code (Sect. 3), and then explore the impact on the cooling times of different physical processes (Sect. 3). Conclusions are given in Sect. 4.

\section{Numerical tools}

In our calculations, the outer boundary conditions were obtained using the pure-hydrogen LTE model atmospheres described at length in Rohrmann et al. (2001, 2002, 2011). Specifically, we computed pressure, temperature, and outer mass fraction at a Rosseland mean optical depth $\tau_{\text {Ross }}=25.1189\left(\log \tau_{\text {Ross }}=1.4\right)$ for $40000 \mathrm{~K} \leq T_{\mathrm{eff}} \leq 2000 \mathrm{~K}$ and $6.5 \leq \log g \leq 9.5$. Model atmospheres were computed in the range $-6 \leq \log \tau_{\text {Ross }} \leq$ 2 (in steps of $0.1 \mathrm{dex}$ ) assuming hydrostatic and radiativeconvective equilibrium. Convective transport was treated within the usual mixing-length (ML2) approximation, in which the ratio of the mixing-length to the pressure scale height is $\alpha=1$. The microphysics comprises nonideal effects in the gas equation of state and chemical equilibrium based on the occupation probability formalism describe in Rohrmann et al. (2002). The chemical composition of the atmosphere includes $\mathrm{H}, \mathrm{H}_{2}, \mathrm{H}^{+}$, $\mathrm{H}^{-}, \mathrm{H}_{2}^{+}, \mathrm{H}_{3}^{+}, \mathrm{He}, \mathrm{He}^{-}, \mathrm{He}^{+}, \mathrm{He}^{2+}, \mathrm{He}_{2}^{+}, \mathrm{HeH}^{+}$, and $\mathrm{e}^{-}$. The level occupation probabilities are self-consistently incorporated into the calculation of the line and continuum opacities. Collisioninduced absorptions due to $\mathrm{H}_{2}-\mathrm{H}_{2}$ (Borysow et al. 2001), $\mathrm{H}_{2}-\mathrm{H}$ (Gustafsson \& Frommhold 2003), H-H (Doyle 1968), $\mathrm{H}_{2}-\mathrm{He}$ (Jorgensen et al. 2000), and H-He pairs (Gustafsson \& Frommhold 2001) were also taken into account. Model atmospheres explicitly include the Lyman $\alpha$ quasi-molecular opacity as a result of eight allowed electric dipole transitions arising from $\mathrm{H}-\mathrm{H}$ and $\mathrm{H}-\mathrm{H}_{2}$ collisions (Rohrmann et al. 2011). This opacity reduces the predicted flux at wavelength $\lambda<4000 \AA$ for stars cooler than $T_{\mathrm{eff}} \approx 6000 \mathrm{~K}$.

\section{Results}

The evolutionary calculations reported here used the LPCODE stellar evolutionary code (Althaus et al. 2003, 2005, 2012). This code was recently used to perform very accurate evolutionary calculations - see García-Berro et al. (2010), Althaus et al. (2010b), Renedo et al. (2010), and references therein. Of relevance for this work, outer boundary condition to the stellar structure and evolution equations are specified by performing three envelope integrations from starting values, as given by the adopted model atmosphere, inward to a fitting outer mass fraction, as described in Kippenhahn et al. (1967). Energy sources resulting from crystallization, the release of latent heat and of energy resulting from carbon-oxygen phase separation, are taken into account using the phase diagram of Horowitz et al. (2010), see Althaus et al. (2012) for details. The equation of state is that of Segretain et al. (1994) for the high-density regime, which accounts for all the important contributions for both the liquid and solid phases (Althaus et al. 2007), complemented by an updated version of the equation of state of Magni \& Mazzitelli (1979) for the low-density regime. Radiative opacities are those of OPAL (Iglesias \& Rogers 1996), including carbon- and oxygen-rich compositions, complemented with the low-temperature opacities of Ferguson et al. (2005), linearly extrapolated to high densities when needed. Conductive opacities were taken from Cassisi et al. (2007). We also consider the effects of element diffusion due to gravitational settling, chemical and thermal diffusion of ${ }^{1} \mathrm{H},{ }^{3} \mathrm{He},{ }^{4} \mathrm{He},{ }^{12} \mathrm{C},{ }^{13} \mathrm{C},{ }^{14} \mathrm{~N}$, and ${ }^{16} \mathrm{O}$, see Althaus et al. (2003) for details. In particular, the metal mass fraction $Z$ in the envelope of our models is specified by scaling it to the local abundance of the CNO elements at each layer. To account for this, we consider radiative opacities tables from OPAL for arbitrary metallicities. Convection is treated within the ML2 version ( $\alpha=1$ ) of the mixing-length theory.

We computed the evolution of sequences of white dwarfs of 0.6 and $0.9 M_{\odot}$. Initial configurations were the result of the complete evolution of 1.75 and $5.0 M_{\odot}$ progenitors, respectively, with metallicity $Z=0.01$ (see Renedo et al. 2010, for details). Progenitor stars were evolved from the zero-age main sequence, through the thermally-pulsing and mass-loss phases on the asymptotic giant branch, to the cooling phase. Time-dependent overshoot mixing beyond the formal convective boundary during the core hydrogen- and helium-burning stages was taken into account, see Althaus et al. (2005) for details. Mass loss during the RGB and AGB phases was considered following the prescription of Schröder \& Cuntz (2005) and Vassiliadis \& Wood (1993), respectively. The outer chemical profiles of our sequences are the result of element diffusion processes that lead to the formation of pure hydrogen envelopes with zero metallicity. The total mass of hydrogen left after hydrogen burning is completely exhausted amounts to $7 \times 10^{-5} M_{\odot}$ for the $0.6 M_{\odot}$ and $7.6 \times 10^{-6} M_{\odot}$ for the $0.9 M_{\odot}$ sequences. For each stellar mass, we computed the cooling phase down to very low luminosities, when most of the white dwarf had already crystallized.

Standard outer boundary conditions are usually based on the Eddington gray approximation, which assumes the diffusion approximation for radiative transfer and neglects convection at low optical depths lower than $\tau_{\text {Ross }}=2 / 3$. A simple test for the diffusion approximation is to compare the ratio between the second $\left(K_{v}\right)$ and zero-order $\left(J_{v}\right)$ moments of the radiation field, with its asymptotic value $(1 / 3)$ at large depth $\left(\tau_{\text {Ross }} \rightarrow \infty\right.$ ). Figures 1 and 2 show such comparison at optical depths $10^{-4.2}<\tau_{\text {Ross }}<10^{2}$ for detailed, pure hydrogen atmospheres with $\log g=8$ and $2000 \mathrm{~K}<T_{\text {eff }}<19000 \mathrm{~K}$, and for $\lambda=2000$ and $5000 \AA$, respectively. In general, the difference between $K_{v} / J_{v}$ and $1 / 3$ increases outward, first with negative values and then with positive ones, forming a complex pattern in the plane $\left(T_{\text {eff }}, \log \tau_{\text {Ross }}\right)$. Departures from the diffusion limit at $\tau_{\text {Ross }}=2 / 3\left(\log \tau_{\text {Ross }}=-0.176\right)$ become larger than $1-5 \%$ depending on the wavelength and effective temperature. Large deviations occur mainly for the visible (Fig. 2) and infrared wavelengths at low $T_{\text {eff }}$. This depth, therefore, is not optimal for establishing the outer boundary conditions of cool white dwarfs.

Convective transport represents another serious limitation of the standard method to evaluate boundary conditions. Figure 3 shows the fraction of the energy flux carried out by convection as a function of $T_{\text {eff }}$ in model atmospheres with $\log g=8$. A superficial convection zone starts at $T_{\text {eff }} \approx 18000 \mathrm{~K}$ associated with the recombination of hydrogen. Below $T_{\text {eff }} \approx 12200 \mathrm{~K}$, the efficiency of convection increases rapidly with depth and the convection zone extends down to the bottom of the atmosphere $\left(\tau_{\text {Ross }}=100\right)$. When the star cools below $T_{\text {eff }} \approx 7000 \mathrm{~K}$, the top of the convection zone slowly extends to very low optical depths as a result of $\mathrm{H}_{2}$ formation. Convection efficiency declines for models cooler than $T_{\mathrm{eff}} \approx 4000 \mathrm{~K}$. It is clear that 
R. D. Rohrmann et al.: Boundary conditions for evolving cool white dwarfs $(R N)$

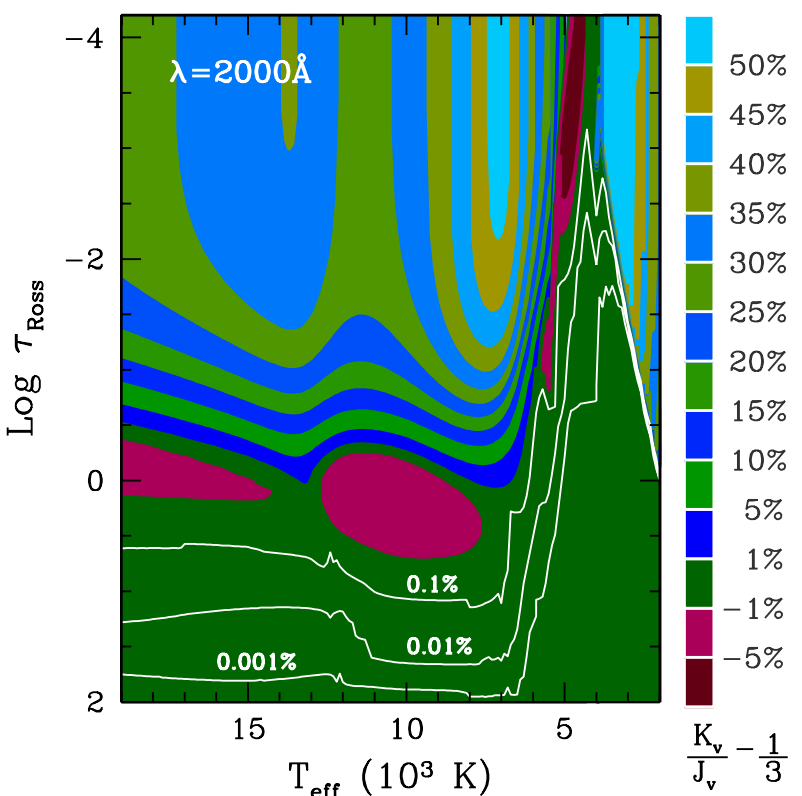

Fig. 1. Test of the diffusion approximation in the plane $\left(T_{\text {eff }}, \log \tau_{\text {Ross }}\right)$ based on differences between the ratio $K_{v} / J_{v}$ and its asymptotic value $1 / 3$ at large optical depth, for a wavelength $\lambda=2000 \AA$. These results correspond to detailed non-gray models for $\mathrm{H}$ atmospheres at $\log g=8$.

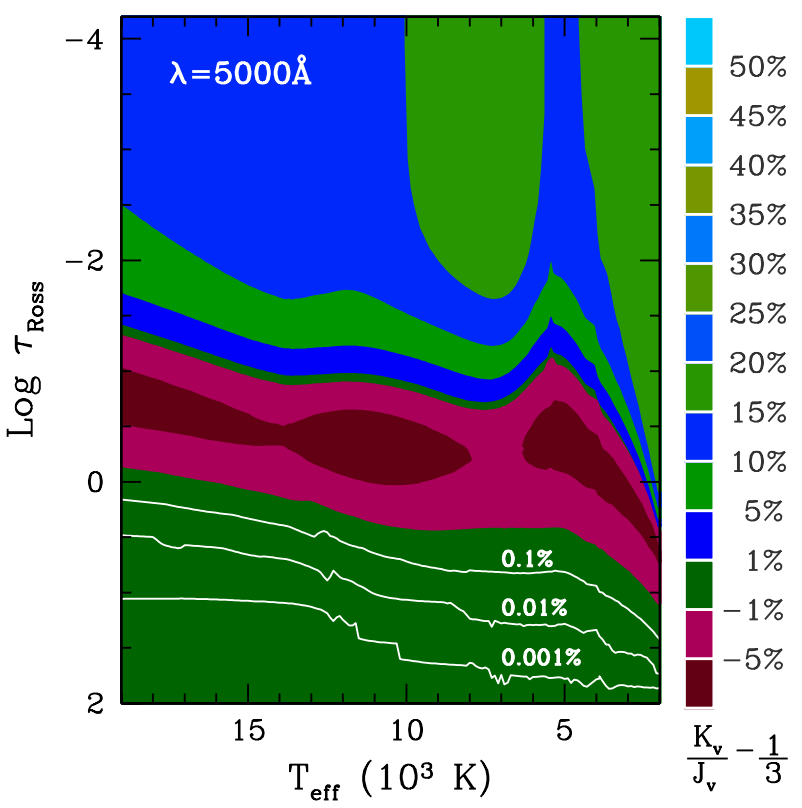

Fig. 2. Same as Fig. 1, but for $\lambda=5000 \AA$.

for $3000 \mathrm{~K} \lesssim T_{\text {eff }} \lessgtr 12500 \mathrm{~K}$, convection may carry more than $30-40 \%$ of the total flux at $\tau_{\text {Ross }} \lesssim 2 / 3$.

The importance of the gray approximation throughout the atmosphere may be tested by comparing two mean opacities with different spectral weightings, for instance, the Planck ( $\kappa_{\text {Planck }}$ ) and the Rosseland ( $\kappa_{\text {Ross }}$ ) means (Mihalas 1978). All mean opacities are equal in a gray atmosphere. Figure 4 displays $\kappa_{\text {Planck }}$ and $\kappa_{\text {Ross }}$ for a hydrogen gas as a function of the temperature for several densities. At high $(\log T \gtrsim 4)$ and $\operatorname{low}(\log T \lesssim$ $3.5)$ temperatures, the opacity is mainly due to atomic and molecular hydrogen, respectively, both yielding large discrepancies between $\kappa_{\text {Planck }}$ and $\kappa_{\text {Ross }}$, and therefore strong deviations from the gray approximation. At intermediate temperatures

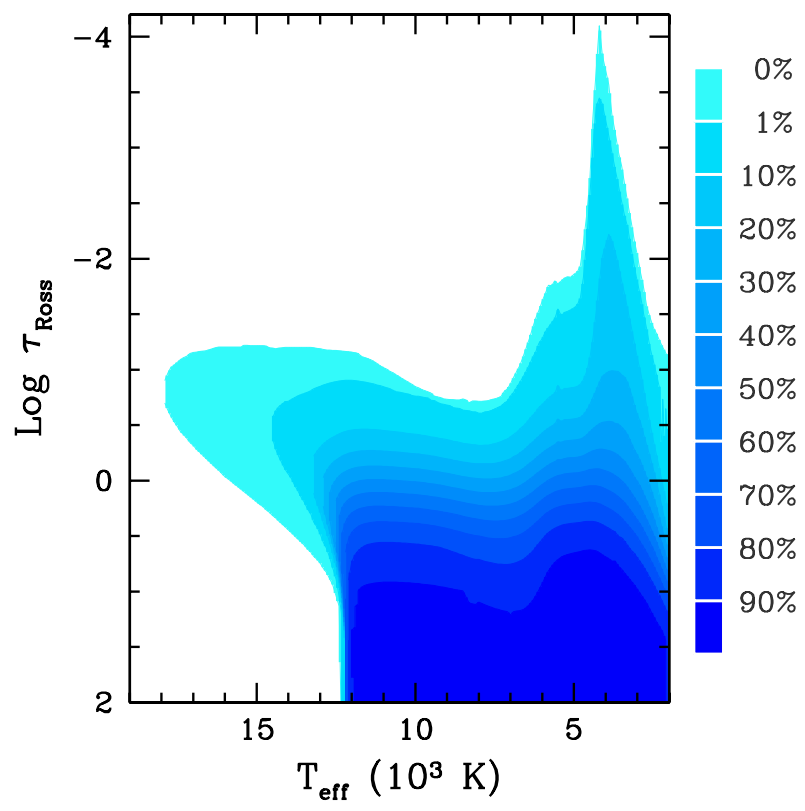

Fig. 3. Fraction of convective energy in the plane $\left(\log \tau_{\text {Ross }}, T_{\text {eff }}\right)$ for model atmospheres with $\log g=8$.

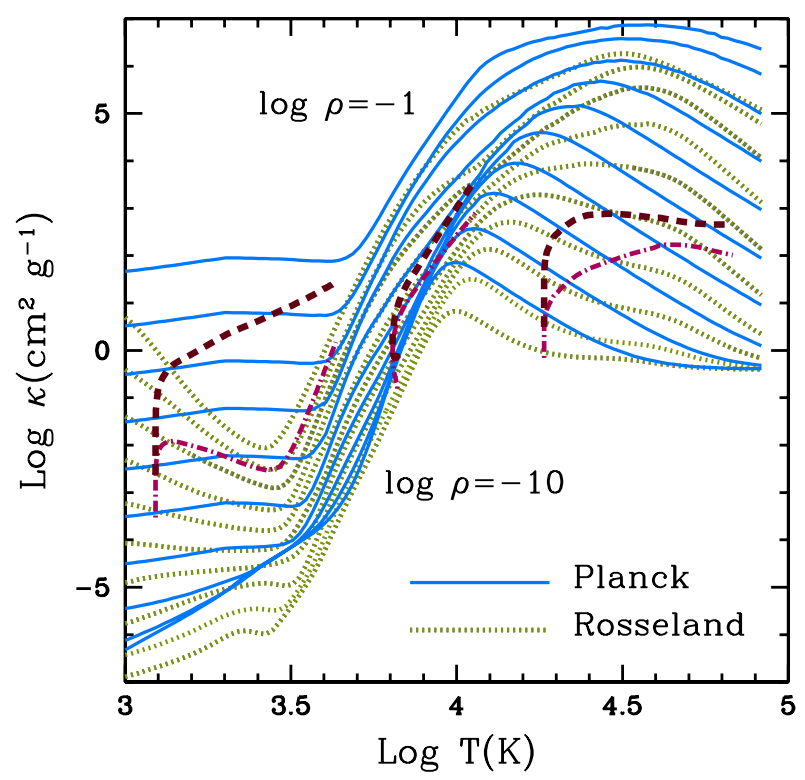

Fig. 4. Planck (solid lines) and Rosseland (dotted lines) mean opacities as a function of the temperature at mass densities increasing from $\log \rho=-10$ to $\log \rho=-1$ in steps of one dex (from bottom to top). Thick dashed and dashed-dotted curves are the Planck and Rosseland mean opacities for white dwarf atmospheres with $\log g=8$ and $T_{\text {eff }}=$ $3000 \mathrm{~K}$ (left), $8000 \mathrm{~K}$ (middle), and $30000 \mathrm{~K}$ (right).

( $3.5 \lesssim \log T \lesssim 4$ ), the differences between the two mean opacities are smaller because the $\mathrm{H}^{-}$absorption, which is the dominant opacity source, has a nearly flat behavior in part of the spectrum. Figure 4 also shows the run of $\kappa_{\text {Planck }}$ and $\kappa_{\text {Ross }}$ for $\mathrm{H}$ atmospheres with $\log g=8$ and $T_{\text {eff }}=3000 \mathrm{~K}, 8000 \mathrm{~K}$, and $30000 \mathrm{~K}$ (from left to right). As expected, the model with $T_{\text {eff }}=8000 \mathrm{~K}$ shows moderate differences between both opacities $(\approx 0.5 \mathrm{dex})$. Discrepancies in the hot model $\left(T_{\mathrm{eff}}=\right.$ $30000 \mathrm{~K})$ are somewhat larger $(0.6-1.2 \mathrm{dex})$, but the largest ones $(1-3$ dex $)$ occur in the cooler model $\left(T_{\text {eff }}=3000 \mathrm{~K}\right)$, which strongly deviates from the gray approximation. 


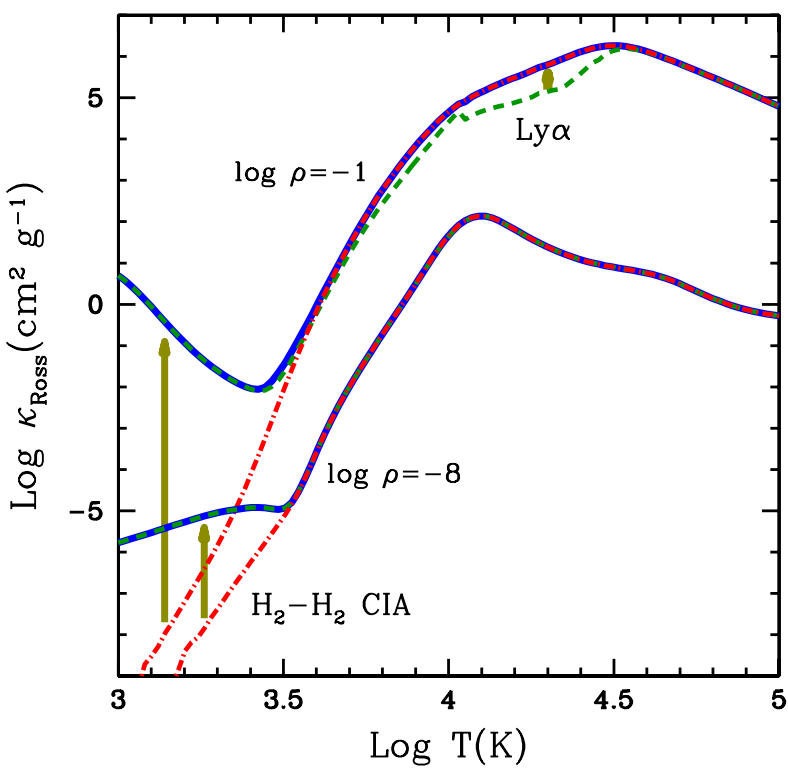

Fig. 5. Effects of the collision-induced opacities $\left(\mathrm{H}_{2}-\mathrm{H}_{2}\right.$ CIA and $\mathrm{Ly} \alpha$ red wing) on the Rosseland mean opacity for two densities, $\log \rho=$ -1 and $\log \rho=-8$. The dashed and dashed-dotted lines correspond respectively to calculations in which the contributions of the Ly $\alpha$ red wing and the $\mathrm{H}_{2}-\mathrm{H}_{2}$ CIA opacities have been removed.

The effects of quasi-molecular processes on the Rosseland mean opacity are illustrated in Fig. 5 for $\log \rho=-8$ and -1 . While the Ly $\alpha$ opacity affects the energy distribution emitted by white dwarfs cooler than $T_{\text {eff }} \approx 5000 \mathrm{~K}$ (Kowalski \& Saumon 2006; Rohrmann et al. 2011), it has a moderate effect on the Rosseland opacity at low densities and relatively high temperatures (see Fig. 5), when the convective coupling with stellar core has not yet occurred. In contrast, abrupt changes in $\kappa_{\text {Ross }}$ occur at low temperatures due to the $\mathrm{H}_{2}-\mathrm{H}_{2}$ CIA processes, which increase the mean opacity by several orders of magnitude and have strong effects on the cooling rates of old white dwarfs (Hansen 1999).

In view of the previous remarks, it seems important to treat the boundary conditions as accurately as possible. Detailed nongray model atmospheres were computed to provide surface boundary conditions for white dwarfs with hydrogen envelopes. The values of the pressure, temperature, and outer fraction of stellar mass were obtained at $\tau_{\text {Ross }}=25.1189\left(\log \tau_{\text {Ross }}=1.4\right)$ for effective temperatures ranging from 2000 to $40000 \mathrm{~K}$ in steps of $100 \mathrm{~K}$, and $\log g$ from 6.5 to 9.5 in steps of 0.1 dex. At $\tau_{\text {Ross }}>$ 25 , the diffusion approximation is guaranteed within $0.01 \%$ or better for most of the spectrum (see Figs. 1 and 2).

To explore the influence of the boundary conditions on the cooling, Figs. 6 and 7 display the relationship between the surface luminosity and age for the 0.6 and $0.9 M_{\odot}$ sequences, respectively, that result from nongray model atmospheres, gray model atmosphere and gray model atmospheres in which the convection is neglected. These figures also show results obtained with boundary conditions at $\tau_{\text {Ross }}=2 / 3$ based on the Eddington gray approximation. Clearly, the use of detailed outer boundary conditions becomes relevant for cooling once evolution has proceeded to luminosities lower than $\log \left(L / L_{\odot}\right) \simeq-3.8(-4.0)$ in the case of $0.60 M_{\odot}\left(0.90 M_{\odot}\right)$ models. These luminosities correspond to effective temperatures lower than $5800 \mathrm{~K}(6100 \mathrm{~K})$. Such values indicate the onset of the convective coupling between the outer envelope and the isothermal degenerate core

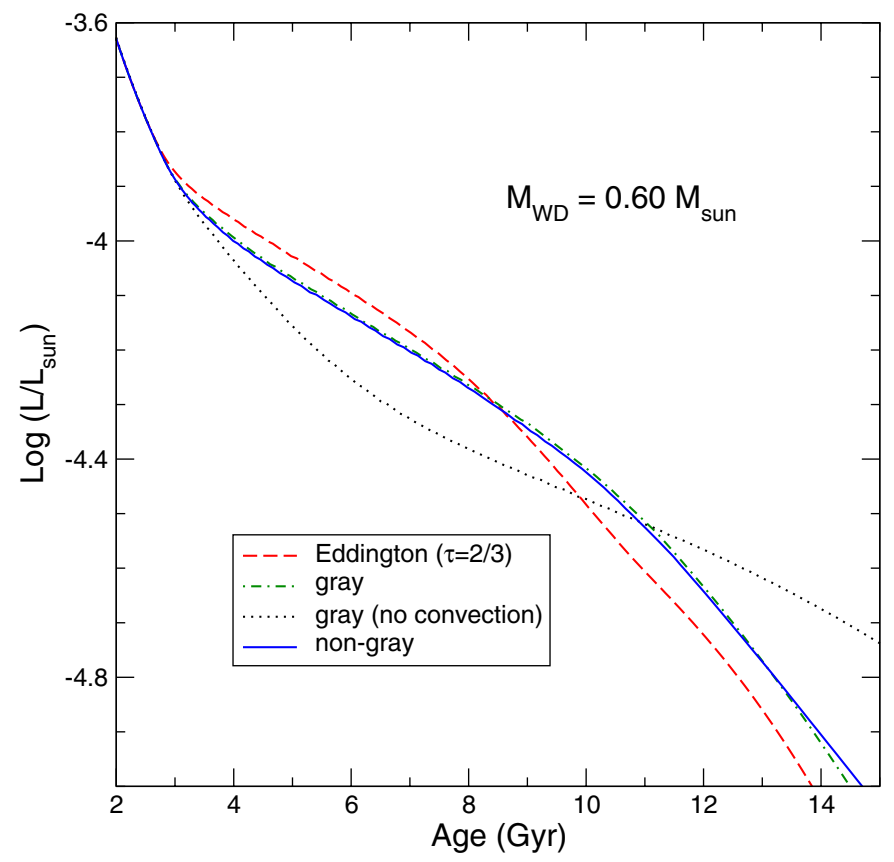

Fig. 6. Surface luminosity versus age for the $0.6 M_{\odot}$ cooling sequences resulting from using outer boundary conditions as given by nongray model atmospheres (solid blue line), gray model atmospheres (dotteddashed green line), and gray model atmospheres in which convection is neglected (dotted line). Results based on the Eddington gray approximation are shown with a dashed red line.

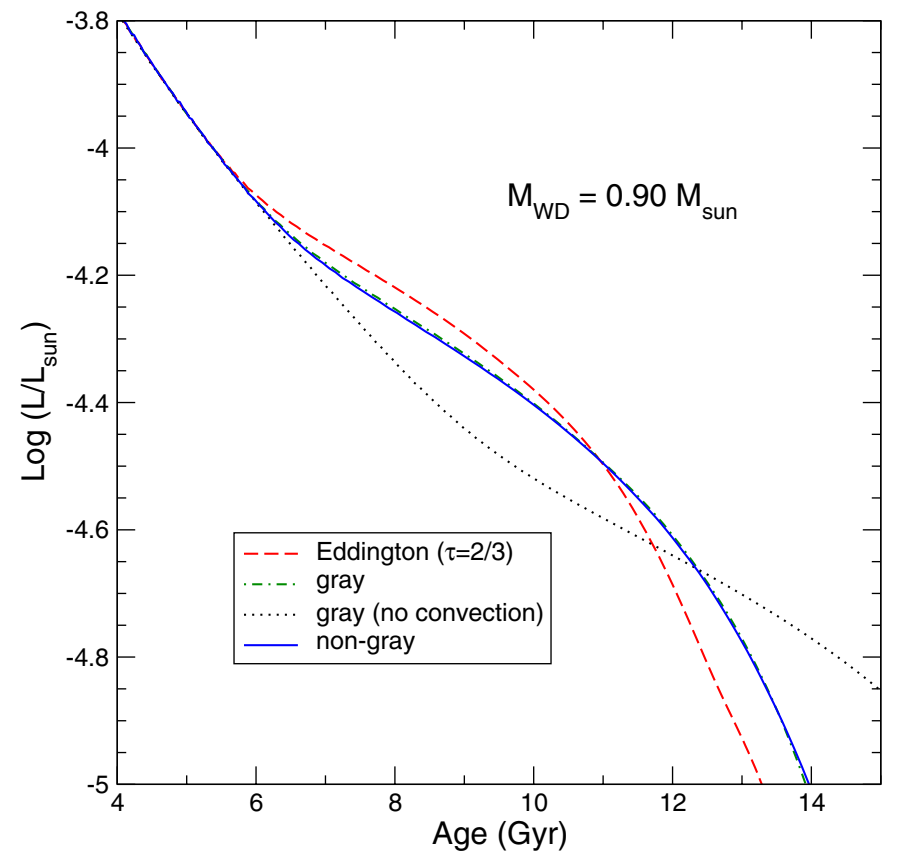

Fig. 7. Same as in Fig. 6 for the $0.9 M_{\odot}$ sequences.

(Tassoul et al. 1990; D’Antona \& Mazzitelli 1990; Prada Moroni $\&$ Straniero 2007). For higher effective temperatures, evolution is almost insensitive to a detailed treatment of the outer boundary conditions.

Figures 6 and 7 show using detailed model atmospheres directly translates into different cooling times from those predicted by the standard Eddington approximation. For the $0.6 M_{\odot}$ sequence, in the range $-3.8 \gtrsim \log \left(L / L_{\odot}\right) \gtrsim-4.3$, including proper outer boundary conditions decreases the cooling ages by up to 
R. D. Rohrmann et al.: Boundary conditions for evolving cool white dwarfs $(R N)$
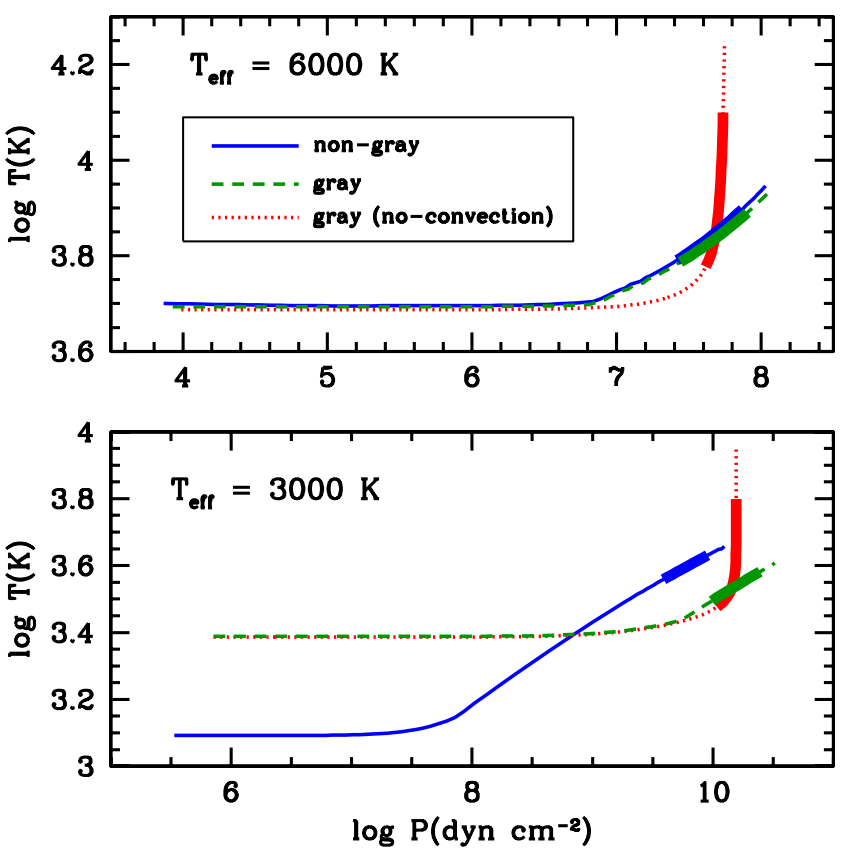

Fig. 8. Temperature-pressure stratifications of pure hydrogen atmospheres with $T_{\text {eff }}=3000 \mathrm{~K}$ and $6000 \mathrm{~K}(\log g=8)$, for different assumptions as indicated in the plot. The top and bottom layers of each model are located at $\tau_{\text {Ross }}=10^{-6}$ and $\tau_{\text {Ross }}=100$, respectively. The thick lines show the layers located between $\tau_{\text {Ross }} \approx 2 / 3$ and $\tau_{\text {Ross }} \approx 25$.

0.7 Gyr, while this trend in the cooling times is reversed at luminosities below $\log \left(L / L_{\odot}\right) \simeq-4.3$, where the use of detailed model atmospheres results in longer cooling times. The behavior of the cooling times is qualitatively similar for the more massive sequence. Again, using any Eddington-like approximation that involves the diffusion assumption for radiative transfer and neglects convection at low optical depths, incorrectly predicts the evolution of cool white dwarfs.

Surprisingly, and contrary to what was expected from Fig. 4, Figs. 6 and 7 show no appreciable changes in the cooling times when the gray approximation is assumed in the model atmospheres. In these calculations the monochromatic opacity coefficient was forced to take the value of the Rosseland mean opacity. In fact, Fig. 8 shows that at the onset of convective coupling $\left(T_{\text {eff }} \approx 6000 \mathrm{~K}\right.$ ) the temperature-pressure stratifications of gray and nongray models are practically identical. Below $T_{\text {eff }} \approx 5000 \mathrm{~K}, \mathrm{H}_{2}-\mathrm{H}_{2}$ collision-induced opacity reduces the surface temperature and increases the temperature in deep atmospheric layers with respect to the gray model. These nongray effects increase towards $T_{\text {eff }} \approx 3000 \mathrm{~K}$ (Fig. 8), which corresponds to about $\log \left(L / L_{\odot}\right) \simeq-4.95$ for the $0.6 M_{\odot}$ sequence (see Fig. 6), but nevertheless have little consequence on the cooling times at observable luminosities. Indeed, they are prominent at very low $T_{\text {eff }}$, and are expected to influence the cooling times once evolution has proceeded to luminosities lower than $\log \left(L / L_{\odot}\right) \simeq-4.95$. We also computed cooling sequences where the collision-induced broadening of $\operatorname{Ly} \alpha$ was omitted in model atmospheres, and we found that this opacity source does not affect the evolution substantially. These results show that processes that markedly alter the distribution of spectral energy radiated by the star may have no effect on its cooling time.

In contrast, neglecting convective energy transfer in model atmospheres strongly alters the cooling times. As illustrated in Fig. 8, neglecting convection results in much higher temperatures at the base of the atmosphere, thus producing a markedly
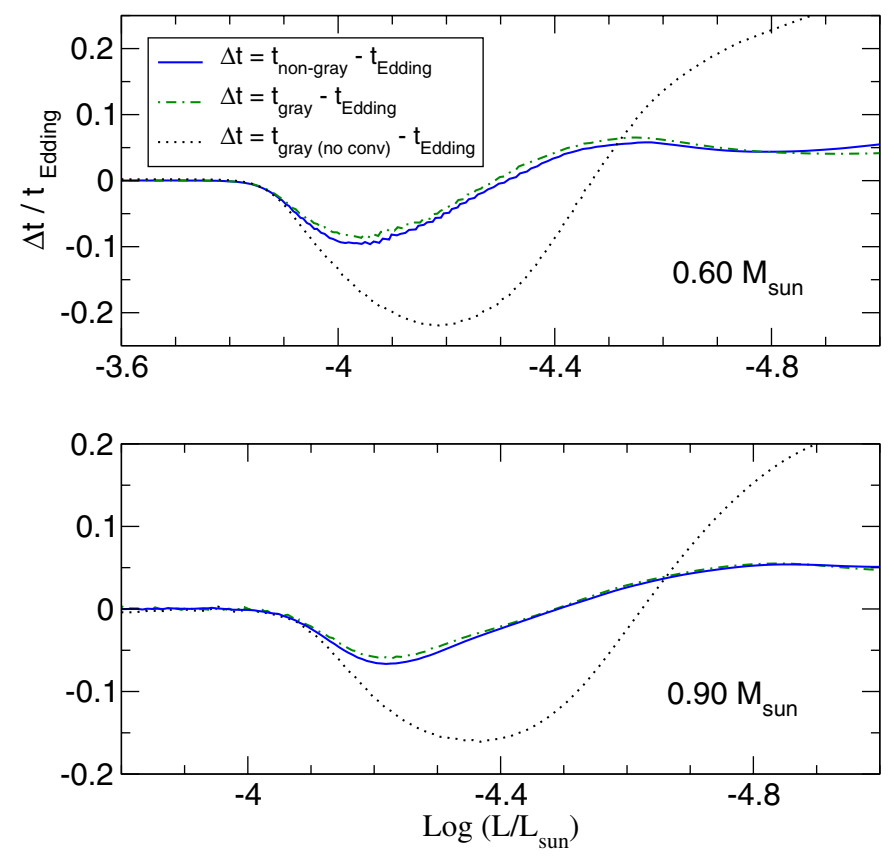

Fig. 9. Age differences between sequences that consider nongray model atmospheres (solid blue line), gray model atmospheres (dotted-dashed green line), and gray model atmospheres in which convection is neglected (dotted line) with respect to the case where the Eddington gray approximation is considered. The upper and bottom panels correspond, respectively, to the 0.6 and $0.9 M_{\odot}$ white dwarf sequences.

shallower outer convection zone, and eventually resulting in age differences of up to 2 Gyr. In particular, for a $0.6 M_{\odot}$ model at $\log \left(L / L_{\odot}\right)=-4$, the use of outer boundary conditions derived from model atmospheres that neglect convection down to an optical depth of $\tau_{\text {Ross }} \approx 25$ leads to an outer convective zone with mass $\log \left(M_{\text {conv }} / M_{\mathrm{WD}}\right) \sim-18$, whereas the value resulting when convection is considered is -13.5 .

The impact on the cooling times of the different boundary conditions can be appreciated better by inspecting Fig. 9, which illustrates the age differences with respect to the case in which the Eddington gray approximation is used for sequences that consider non-gray model atmospheres, gray model atmospheres, and gray model atmospheres in which convection is neglected. The upper and bottom panels correspond to the 0.6 and $0.9 M_{\odot}$ sequences, respectively. For the $0.6 M_{\odot}$ sequence, age differences are negative in the range $-3.8 \gtrsim \log \left(L / L_{\odot}\right) \gtrsim-4.3$ and using detailed model atmospheres predicts ages that are up to $\approx 10 \%$ shorter at $\log \left(L / L_{\odot}\right)=-4.05$ when compared with the ages derived using the Eddington approximation. The differences are somewhat smaller for the $0.9 M_{\odot}$ sequence, reaching up to $\approx 7 \%$ at $\log \left(L / L_{\odot}\right)=-4.2$. Besides this, the gray assumption translates into age differences less than $\sim 1 \%$ in both sequences.

Since the input physics adopted in the codes used to compute the stellar interior and the atmosphere are not exactly the same, we examined the impact of matching atmosphere and interior models at different optical depths. We found that the cooling times differ at most $0.7 \%(0.9 \%)$ for boundary conditions at $\tau_{\text {Ross }} \approx 50$ (100) with respect to those obtained at $\tau_{\text {Ross }} \approx 25$. Thus, differences in the constitutive physics in the codes appear to have small consequences in the derivation of the boundary conditions. We also tested the effects of changing the value of the mixing length parameter $(\alpha)$ in the convection theory. In particular, if the efficiency of convection is increased to $\alpha=2$ 
(ML3 version of the mixing-lenght theory), the relative age differences with respect to the use of $\alpha=1$ (ML2 version) becomes smaller than $0.8 \%(0.4 \%)$ for the $0.6 M_{\odot}\left(0.9 M_{\odot}\right)$ model.

\section{Conclusions}

The purpose of this work has been to provide detailed outer boundary conditions that allow white dwarf evolution to be computed in a consistent way with the predictions of detailed model atmospheres. Data are provided in the form of tables for a wide range of surface gravities and effective temperatures, which are appropriate for computing the evolution of cool white dwarfs with pure hydrogen atmospheres. The full set of data is available at the CDS, at http://www.icate-conicet.gob.ar/ rohrmann/tables.html, or upon request to the authors at their e-mail addresses.

White dwarf cooling timescales are sensitive to the surface boundary conditions for effective temperatures lower than $T_{\text {eff }} \approx 6000 \mathrm{~K}$. Different outer boundary conditions may result in substantial differences in the cooling times for cooler white dwarfs. However, nongray effects do not become important in the cooling rates. On the other hand, depending on the stellar luminosity, the use of detailed model atmospheres like the ones presented here results in age differences of about $10 \%$ when compared with the ages computed using the Eddington approximation, which assumes the diffusion approximation for radiative transfer and neglects convection at low optical depths lower than $\tau_{\text {Ross }}=2 / 3$. These differences are close to the current uncertainties in the white-dwarf cooling times at the low luminosities that result from uncertainties in the treatment of progenitor evolution, particularly during the core helium-burning phase (Prada Moroni \& Straniero 2002; Salaris et al. 2010). Consequently, accurate outer boundary conditions provided by detailed model atmospheres have to be considered in evolutionary studies aimed at using these stars as accurate cosmic clocks.

Acknowledgements. This research was supported by PIP 112-200801-01474 and PIP 112-200801-00940 from CONICET, by MCINN grant AYA201123102, by the European Union FEDER funds, and by the ESF EUROGENESIS project (grant EUI2009-04167)

\section{References}

Althaus, L. G., Serenelli, A. M., Córsico, A. H., \& Montgomery, M. H. 2003, A\&A, 404, 593

Althaus, L. G., Serenelli, A. M., Panei, J. A., et al. 2005, A\&A, 435, 631

Althaus, L. G., García-Berro, E., Isern, J., Córsico, A. H., \& Rohrmann, R. D. 2007, A\&A, 465, 249

Althaus, L. G., Córsico, A. H., Isern, J., \& García-Berro, E. 2010a, A\&ARv, 18, 471
Althaus, L. G., Córsico, A. H., Bischoff-Kim, A., et al. 2010b, ApJ, 717, 897 Althaus, L. G., García-Berro, E., Isern, J., Córsico, A. H., \& Miller Bertolami, M. M. 2012, A\&A, 537, A33

Bergeron, P., Wesemael, F., \& Fontaine, G., 1991, ApJ, 367, 253

Böhm, K. H., \& Grenfell, T. C. 1973, A\&A, 28, 79

Borysow, A., Jorgensen, U. G., \& Fu, Y. 2001, JQSRT, 68, 235

Cassisi, S., Potekhin, A. Y., Pietrinferni, A., Catelan, M., \& Salaris, M. 2007, ApJ, 661, 1094

Córsico, A. H., Benvenuto, O. G., Althaus, L. G., Isern, J., \& García-Berro, E. 2001, New Astron., 6, 197

D’Antona, F., \& Mazzitelli, I. 1990, ARA\&A, 28, 139

Doyle, R. O. 1968, ApJ, 153, 987

Ferguson, J. W., Alexander, D. R., Allard, F., et al. 2005, ApJ, 623, 585

García-Berro, E., Hernanz, M., Isern, J., \& Mochkovitch, R. 1995, MNRAS, 277,801

García-Berro, E., Torres, S., Althaus, L. G., et al. 2010, Nature, 465, 194

García-Berro, E., Lorén-Aguilar, P., Torres, S., Althaus, L. G., \& Isern, J. 2011, J. Cosmol. Astropart., 5, 21

Gustafsson, M., \& Frommhold, L. 2001, ApJ, 546, 1168

Gustafsson, M., \& Frommhold, L. 2003, A\&A, 400, 1161

Hansen, B. M. S. 1998, Nature, 394, 869

Hansen, B. M. S. 1999, ApJ, 520, 680

Hansen, B. M. S., Anderson, J., Brewer, J., et al. 2007, ApJ, 671, 380

Horowitz, C. J., Schneider, A. S., \& Berry, D. K. 2010, Phys. Rev. Lett., 104, 231101

Iglesias, C. A., \& Rogers, F. J. 1996, ApJ, 464, 943

Isern, J., Hernanz, M., \& García-Berro, E. 1992, ApJ, 392, L23

Isern, J., García-Berro, E., Torres, S., \& Catalán, S. 2008, ApJ, 682, L109

Jorgensen, U. G., Hammer, D., Borysow, A., \& Falkesgaard, J. 2000, A\&A, 361, 283

Kippenhahn, R., Weigert, A., \& Hofmeister, E. 1967, Methods in computational physics, eds. B. Alder, S. Fernbach, \& M. Rottenberg (New York: Academic Press), 7

Kowalski, P., \& Saumon, D. J. 2006, ApJ, 651, L137

Magni, G., \& Mazzitelli, I. 1979, A\&A, 72, 134

Mestel, L. 1952, MNRAS, 112, 583

Mihalas, D. 1978, Stellar atmospheres, 2nd. edn. (San Francisco: Freeman)

Prada Moroni, P. G., \& Straniero, O. 2002, ApJ, 581, 585

Prada Moroni, P. G., \& Straniero, O. 2007, A\&A, 466, 1043

Renedo, I., Althaus, L. G., Miller Bertolami, M. M., et al. 2010, ApJ, 717, 183

Rohrmann, R. D. 2001, MNRAS, 323, 699

Rohrmann, R. D., Serenelli, A. M., Althaus, L. G., \& Benvenuto, O. G. 2002, MNRAS, 335, 499

Rohrmann, R. D., Althaus, L. G., \& Kepler, S. O. 2011, MNRAS, 411, 781

Salaris, M., García-Berro, E., Hernanz, M., Isern, J., \& Saumon, D. 2000, ApJ, 544, 1036

Salaris, M., Cassisi, S., Pietrinferni, A., Kowalski, P. M., \& Isern, J. 2010, ApJ, 716,1241

Saumon, D., \& Jacobson, S. B. 1999, ApJ, 511, L107

Schröder, K.-P., \& Cuntz, M. 2005, ApJ, 630, L73

Segretain, L., Chabrier, G., Hernanz, M., et al. 1994, ApJ, 434, 641

Serenelli, A. M., Althaus, L. G., Rohrmann, R. D., \& Benvenuto, O. G. 2001, MNRAS, 325, 607

Tassoul, M., Fontaine, G., \& Winget, D. E. 1990, ApJS, 72, 335

Vassiliadis, E., \& Wood, P. R. 1993, ApJ, 413, 641

von Hippel, T., \& Gilmore, G. 2000, AJ, 120, 1384

Winget, D. E., Sullivan, D. J., Metcalfe, T. S., Kawaler, S. D., \& Montgomery, M. H. 2004, ApJ, 602, L109

Winget, D. E., Kepler, S. O., Campos, F., et al. 2009, ApJ, 693, L6 